\title{
Imaging with pairs of skew lenses
}

Jakub Bělín, Stephen Oxburgh, Tomáš Tyc, Johannes Courtial

Jakub Bělín, Stephen Oxburgh, Tomáš Tyc, Johannes Courtial, "Imaging with pairs of skew lenses," Proc. SPIE 10376, Novel Optical Systems Design and Optimization XX, 1037607 (24 August 2017); doi: 10.1117/12.2272989

SPIE Event: SPIE Optical Engineering + Applications, 2017, San Diego, California, United States 


\title{
Imaging with pairs of skew lenses
}

\author{
Jakub Bělín ${ }^{\mathrm{a}}$, Stephen Oxburgh ${ }^{\mathrm{a}}$, Tomáš Tyc ${ }^{\mathrm{b}}$, and Johannes Courtial ${ }^{\mathrm{a}}$

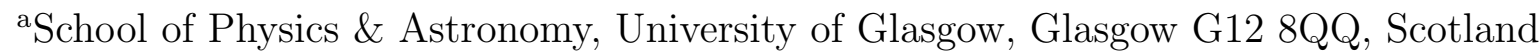 \\ b Institute of Theoretical Physics and Astrophysics, Masaryk University, Kotlarska 2, 61137 \\ Brno, Czech Republic
}

\begin{abstract}
Many of the properties of thick lenses can be understood by considering these as a combination of parallel ideal thin lenses that share a common optical axis. A similar analysis can also be applied to many other optical systems. Consequently, combinations of ideal lenses that share a common optical axis, or at least optical-axis direction, are very well understood. Such combinations can be described as a single lens with principal planes that do not coincide. However, in recent proposals for lens-based transformation-optics devices the lenses do not share an optical-axis direction. To understand such lens-based transformation-optics devices, combinations of lenses with skew optical axes must be understood.

In complete analogy to the description of combinations of pairs of ideal lenses that share an optical axis, we describe here pairs of ideal lenses with skew optical axes as a single ideal lens with sheared object and image spaces. The transverse planes are no longer perpendicular to the optical axis. We construct the optical axis, the direction of the transverse planes on both sides, and all cardinal points. We believe that this construction has the potential to become a powerful tool for understanding and designing novel optical devices.
\end{abstract}

Keywords: imaging; optical design; lens systems

\section{INTRODUCTION}

Lenses are paraxial imaging elements, and because of their usefulness and ubiquity the imaging properties of lenses are very well understood. The paraxial imaging properties of lenses can be well represented by ideal thin lenses: a single ideal thin lens in the case of a physically thin lens (which has a long focal length), and pairs of parallel ideal* thin lenses - one per surface - in the case of thick lenses.

Combinations of parallel thin lenses can also describe combinations of multiple parallel lenses. Such combinations can be reduced in complexity by describing pairs of parallel lenses as a single thin lens, but one in which the principal planes do not coincide. By successively applying this idea, any number of parallel thin lenses can be reduced to a single thin lens.

Several years ago, we started to study micro-structured, light-ray-direction-changing, sheets called telescope windows or generalised confocal lenslet arrays (GCLAs). ${ }^{1,2}$ Telescope windows are arrays of tiny telescopes; when viewing them from a suitable distance, the individual telescopes cannot be resolved and telescope windows appear to be homogeneous sheets that perform generalised refraction. In a sense, the telescopes act like the pixels of the telescope window, which performs pixellated generalised refraction. Telescope windows have a number of limitations due to their pixellated nature ${ }^{3,4}$ but the pixellation also enables them to perform generalised laws of refraction that would not be possible without pixellation (or another mechanism that introduces phase discontinuities into the transmitted beam). ${ }^{5,6}$ This gives planar telescope windows very powerful imaging prop$\operatorname{erties}^{7,8}$ (note that non-planar refracting interfaces do not posses these imaging properties ${ }^{9}$ ), but the imaging is not stigmatic but integral, which is of lower quality. Specifically, such planar telescope windows can perform the most general imaging that maps all points in object space to corresponding points in image space. Telescope windows with those imaging properties are very closely related to Gabor superlenses. ${ }^{10}$

Further author information: (Send correspondence to J.C.)

J.C.: E-mail: johannes.courtial@glasgow.ac.uk, Telephone: +44 (0)141 3306429

*In the following, we will drop the "ideal" and refer to ideal thin lenses simply as thin lenses.

Novel Optical Systems Design and Optimization XX, edited by Arthur J. Davis,

Cornelius F. Hahlweg, Joseph R. Mulley, Proc. of SPIE Vol. 10376, 1037607

(C) 2017 SPIE · CCC code: $0277-786 X / 17 / \$ 18 \cdot$ doi: $10.1117 / 12.2272989$ 


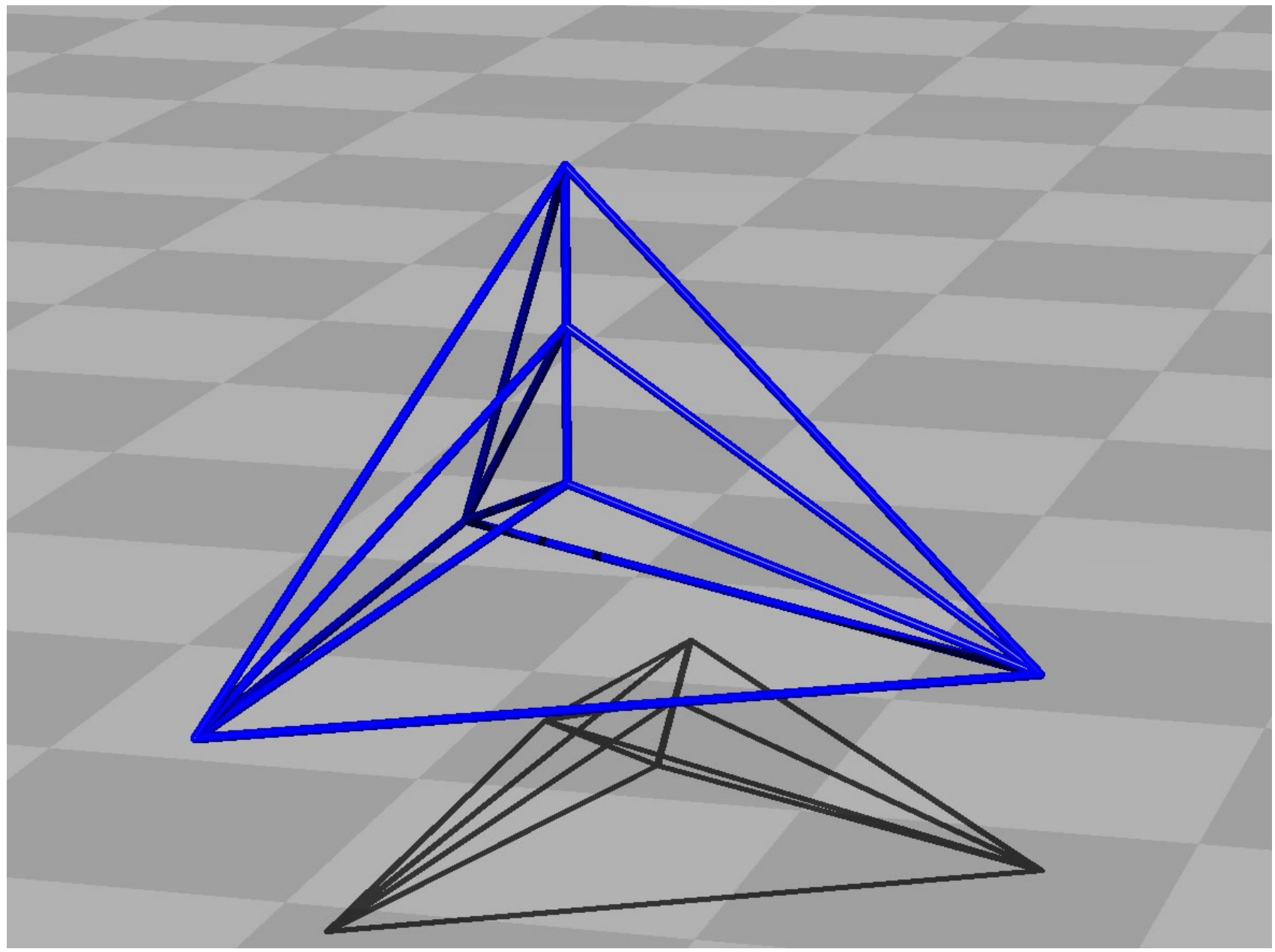

Figure 1. Cylinder-frame model illustrating the structure of an example of a transformation-optics device consisting of ideal thin lenses. The blue cylinders mark the edges of the lenses; every triangle formed by these blue cylinders contains an ideal thin lens. The lenses themselves are not shown. The image was created with an extended version of our custom raytracer Dr TIM. ${ }^{15}$

To search for applications of telescope windows, we idealising them and studied these idealised telescope windows theoretically. The idealisation neglects all negative effects of pixellation; when the actual telescope windows perform integral imaging, then their idealised counterparts perform stigmatic (i.e. ray-optically perfect) imaging. First we investigated the simplest case of idealised homogeneous telescope windows, where the generalised law of refraction is the same at each point of the window. (The non-idealised realisation of such homogeneous telescope windows is one in which all telescopes forming the telescope window are identical.) We learnt how to construct transformation-optics devices from structures of triangular homogeneous telescope windows. ${ }^{11,12}$ Next, we turned our attention to the more complicated case of idealised inhomogeneous telescope windows that image any point in object space to a corresponding point in image space. Such telescope windows are generalisations of ideal thin lenses that have different focal lengths on their two sides; for simplicity, we called such generalised ideal thin lenses glenses. ${ }^{13}$ As before, we learnt how to construct transformation-optics devices from structures of glenses. ${ }^{14}$ Interestingly, in a few of the glenses in the structure the focal lengths on the two sides of the glens were identical, meaning that these were actually ideal thin lenses.

This led to the question "Can we design transformation-optics devices purely from ideal thin lenses?" Elsewhere we answer this question affirmatively by constructing an example of a structure of ideal thin lenses that acts as a transformation-optics device (Fig. 1). The ideal thin lenses that form this structure are very much 


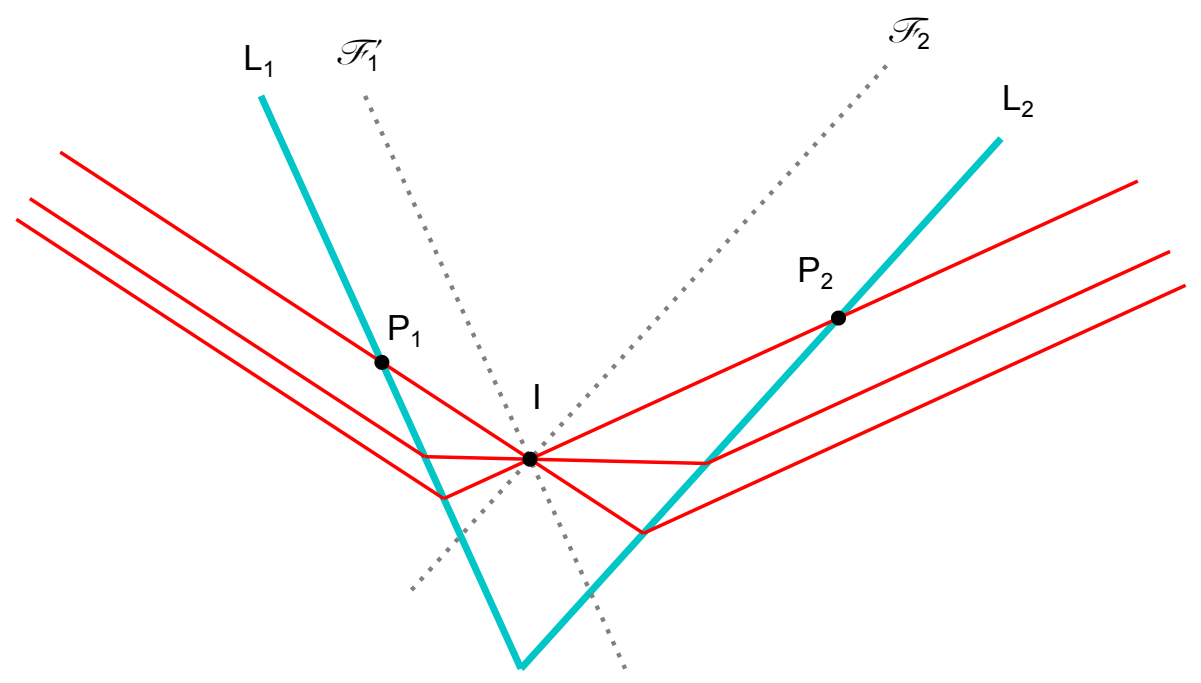

Figure 2. Telescopic character of combinations of skew ideal thin lenses, $\mathrm{L}_{1}$ and $\mathrm{L}_{2}$. The cyan lines marked $\mathrm{L}_{1}$ and $\mathrm{L}_{2}$ indicate the planes of the two lenses; $\mathrm{P}_{1}$ and $\mathrm{P}_{2}$ are the principal points of the individual lenses; $\mathcal{F}_{1}^{\prime}$ is the image-sided focal plane of lens $1, \mathcal{F}_{2}$ is the object-sided focal plane of lens 2 (we consider light rays travelling from left to right). The red lines show a number of light rays passing through the lens combination. The light rays are parallel before passing through $\mathrm{L}_{1}$, which redirects them such that they pass through a point I on the line where the focal planes $\mathcal{F}_{1}^{\prime}$ and $\mathcal{F}_{2}$ intersect, and after transmission through $\mathrm{L}_{2}$ they are parallel again.

non-paraxial in that the lenses traversed by typical light rays are not parallel. There is currently no description of such combinations of skew ideal thin lenses in terms of fewer ideal thin lenses. Like in the case of parallel lenses, such a description would enable a reduction in complexity of problems involving such ideal-thin-lens combinations. However, there are problems that need to be overcome; for example, skew combinations of lenses have the property of transforming certain parallel bundles of rays again into parallel bundles of rays, giving the lens combination the character of a telescope instead of a thin lens (Fig. 2). Also, there is the fundamental problem of how to even define the optical axis etc. of a combination of skew lenses, concepts that are taken for granted when considering combinations of parallel lenses.

Here we explain how to overcome these problems. We define all relevant concepts (e.g. the optical axis) of a combination of skew lenses, and we provide a description of such a combination as a single thin lens. We also discuss the resolution of the mysterious telescopic character of the lens combination.

This paper is structured as follows. In section 2 we review combinations of parallel ideal thin lenses. Section 3 introduces an analogous description of combinations of skew ideal thin lenses. Section 4 provides a brief discussion and conclusions.

\section{REVIEW OF COMBINATIONS OF PARALLEL IDEAL THIN LENSES}

Two parallel thin lenses that share a common optical axis can be described as a single thin lens as follows.

The choice of optical axis of the combination is obvious: it is the same as the optical axis common to both lenses. Clearly, this choice has the property that any point on the optical axis is again imaged to the optical axis.

The focal planes can easily be constructed in the same way in which the focal planes of an individual lens could be found. Throughout this paper, we consider all light rays to travel from left to right. These are the transverse planes (which are perpendicular to the optical axis) in which light rays parallel to the optical axis intersect. Fig. 3 shows light rays that are parallel to the optical axis either on the left of lens $\mathrm{L}_{1}$ or to the right of lens $\mathrm{L}_{2}$. Where the former intersect the optical axis after transmission through both lenses is the position of the image-sided focal point, $\mathrm{F}^{\prime}$; the point where the latter intersects the optical axis before transmission through 


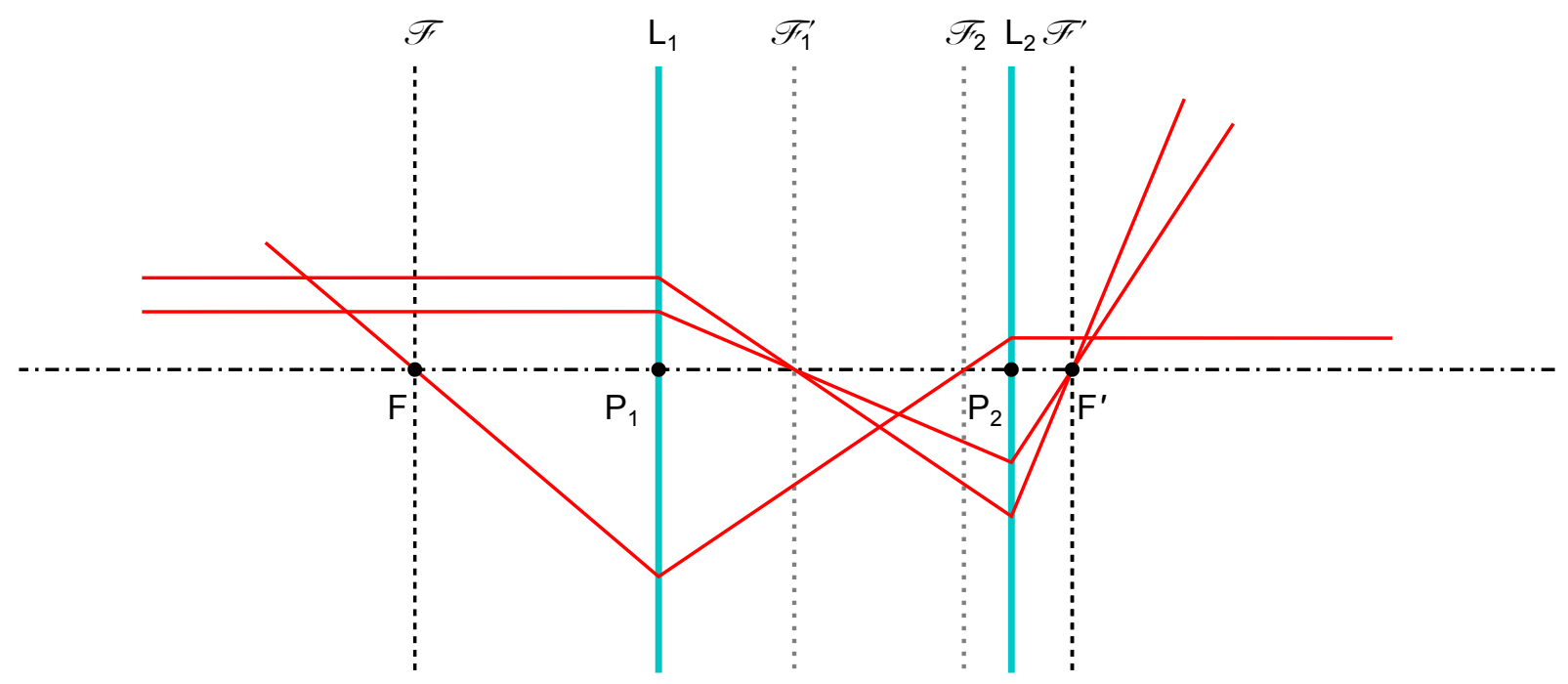

Figure 3. Construction of the focal planes of a combination of parallel ideal thin lenses.

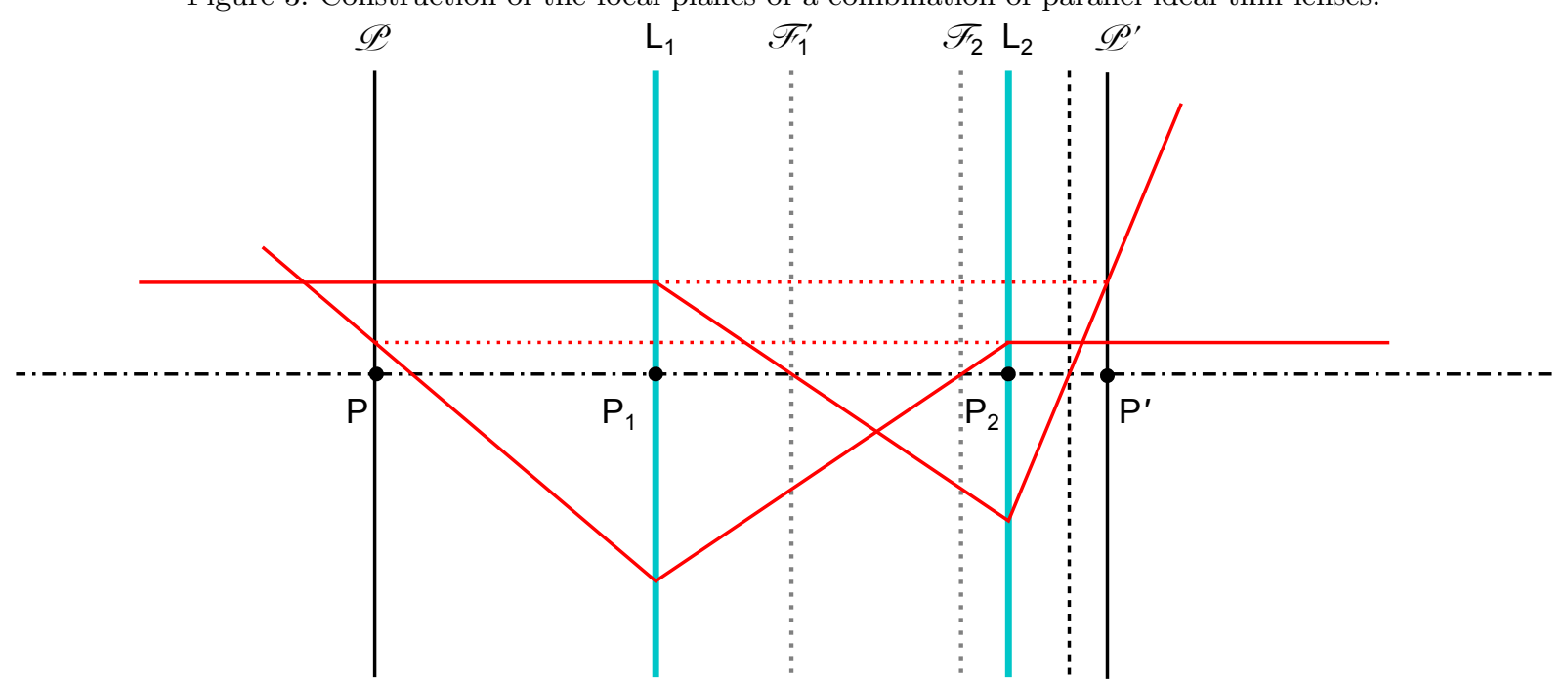

Figure 4. Construction of the principal planes of a combination of parallel ideal thin lenses.

both lenses is the object-sided focal point, F. The object- and image-sided focal planes, $\mathcal{F}$ and $\mathcal{F}^{\prime}$, can then be constructed as the transverse planes through the corresponding focal points.

But the idea that enables the description in terms of a single lens is that of principal planes. In the case of a single thin lens, the principal planes coincide with the lens plane. Light rays in general change direction at this plane, but they enter and exit at at the same position; in other words, the lens plane is imaged into itself with magnification +1 . This is the property that is used to construct the principal planes: when starting with the knowledge (not shown here) that such planes exist and that they are transverse planes, it is easy to show where they have to lie. This is done in Fig. 4, where two light rays, one that is parallel to the optical axis in front of lens $\mathrm{L}_{1}$, the other after behind $\mathrm{L}_{2}$, are traced through the lenses. For the imaging magnification to be +1 , those light rays have to intersect the principal planes on the other side of the lenses at the same height above the optical axis at which they travel when parallel to the optical axis. In this way, it is then easy to construct the object- and image-sided principal planes, $\mathcal{P}$ and $\mathcal{P}^{\prime}$, and the corresponding principal points $\mathrm{P}$ and $\mathrm{P}^{\prime}$ where these planes intersect the optical axis. 


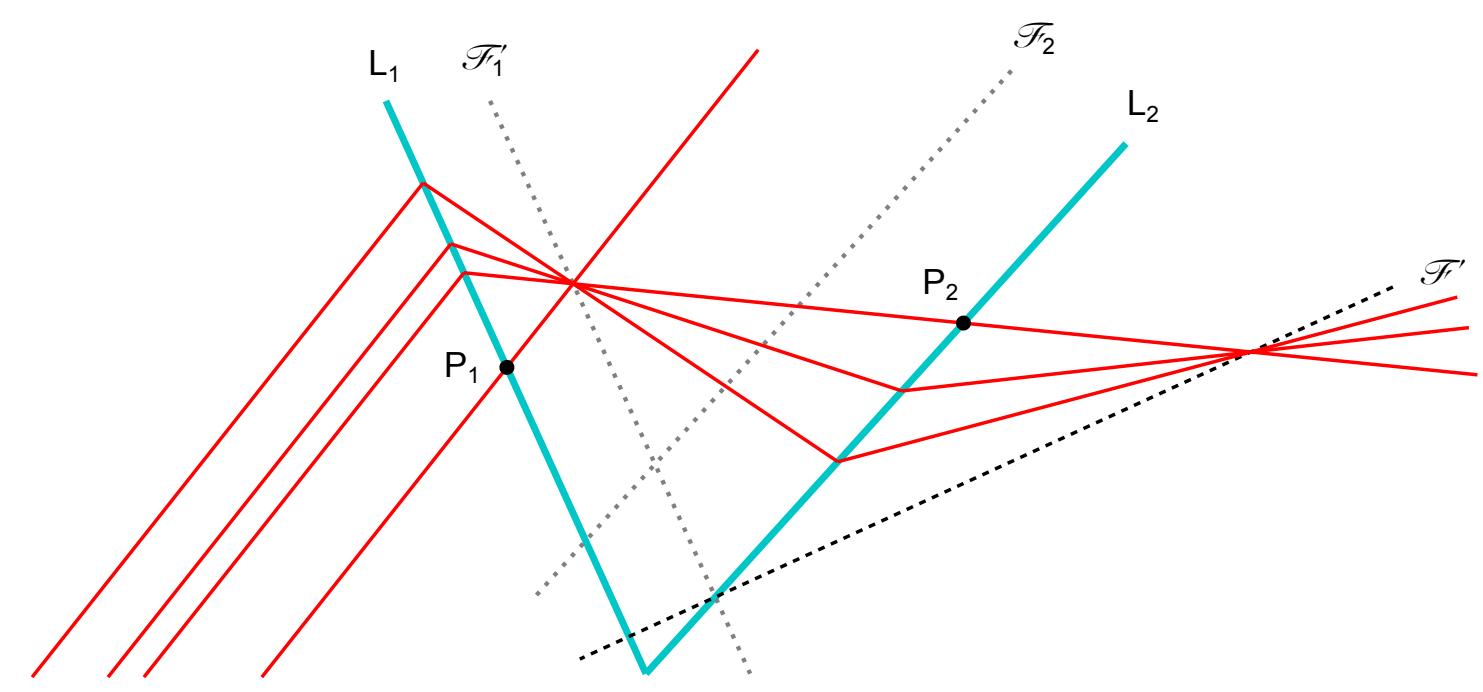

Figure 5. Construction of the image-sided focal plane of the lens combination. Parallel bundles of light rays incident on lens $\mathrm{L}_{1}$ are focussed into a point in $\mathrm{L}_{1}$ 's image-sided focal plane, $\mathcal{F}_{1}^{\prime}$. Lens $\mathrm{L}_{2}$ then images this point into a corresponding point, which lies in the image-sided focal plane $\mathcal{F}^{\prime}$ (black dashed line) of the lens combination. As $\mathrm{L}_{2}$ images any point on the intersection between $\mathcal{F}_{1}^{\prime}$ and $\mathrm{L}_{2}$ to itself, the image-sided focal plane $\mathcal{F}^{\prime}$ must therefore pass through this intersection.

\section{COMBINATIONS OF SKEW IDEAL THIN LENSES}

Any generalisation to skew lenses of the above construction of an individual thin lens that is equivalent to a pair of parallel thin lenses needs to first find suitable generalisations of the concepts of optical axis, focal planes, and principal planes.

The most straightforward of these generalisations, which in fact requires no generalisation at all, is that of the focal planes. These can still be defined as those planes in which bundles of parallel light rays intersect after having passed through both lenses. Fig. 5 shows that the image-sided focal plane $\mathcal{F}^{\prime}$ of the lens combination passes through the line where lens $\mathrm{L}_{1}$ 's image-sided focal plane $\mathcal{F}_{1}^{\prime}$ intersects lens $\mathrm{L}_{2}$. This can be seen as follows. The image-sided focal plane is defined as the plane in which bundles of light rays that are parallel before entering lens $\mathrm{L}_{1}$ intersect after passing through both lenses. As lens $\mathrm{L}_{1}$ on its own focusses such bundles of light rays into a point in its image-sided focal plane $\mathcal{F}_{1}^{\prime}$, and lens $\mathrm{L}_{2}$ then re-images these positions, the focal plane of the combination of both lenses is the image due to $\mathrm{L}_{2}$ of the image-sided focal plane of lens $\mathrm{L}_{1}$. But as $\mathrm{L}_{2}$ images any position on $\mathrm{L}_{2}$ to the same position, it must be true that the image-sided focal plane of the lens combination passes through the line where $\mathcal{F}_{1}^{\prime}$ and $\mathrm{L}_{2}$ intersect. Similarly, the object-sided focal plane $\mathcal{F}$ passes through the line where the object-sided focal plane of lens $\mathrm{L}_{2}, \mathcal{F}_{2}$, intersects lens $\mathrm{L}_{1}$.

Next, we generalise the optical axis. Perhaps the most straightforward of these generalisations defines the optical axis as the line through the principal points of both lenses (Fig. 6).

Finally, we generalise the principal planes. We define these as those transverse planes that pass through the line where the two lenses $\mathrm{L}_{1}$ and $\mathrm{L}_{2}$ themselves intersect (Fig. 7).

But now there is a problem: how do we define the transverse planes? This is a good time to look again at Fig. 2, which shows a bundle of parallel light rays that becomes, after transmission through both lenses, another bundle of parallel light rays. The figure is drawn in 2D, but it is immediately clear that the light rays that pass through any given position on lens $\mathrm{L}_{1}$ and arbitrary points on the line I where the "inner" focal planes (i.e. $\mathcal{F}_{1}^{\prime}$ and $\mathcal{F}_{2}$ ) intersect all lie in the same plane. Light rays that pass through a different point on $\mathrm{L}_{1}$ and arbitrary points on the line I all lie in a different, parallel, plane. These planes are examples of transverse planes in object space, and we define transverse planes in image space analogously. This definition retains the familiar property of all transverse planes in object space to be parallel, and the same is true for all transverse planes in image space, but transverse planes in object space are in general not parallel to those in image space. Also unfamiliar is the fact that the transverse planes are not perpendicular to the optical axis. 


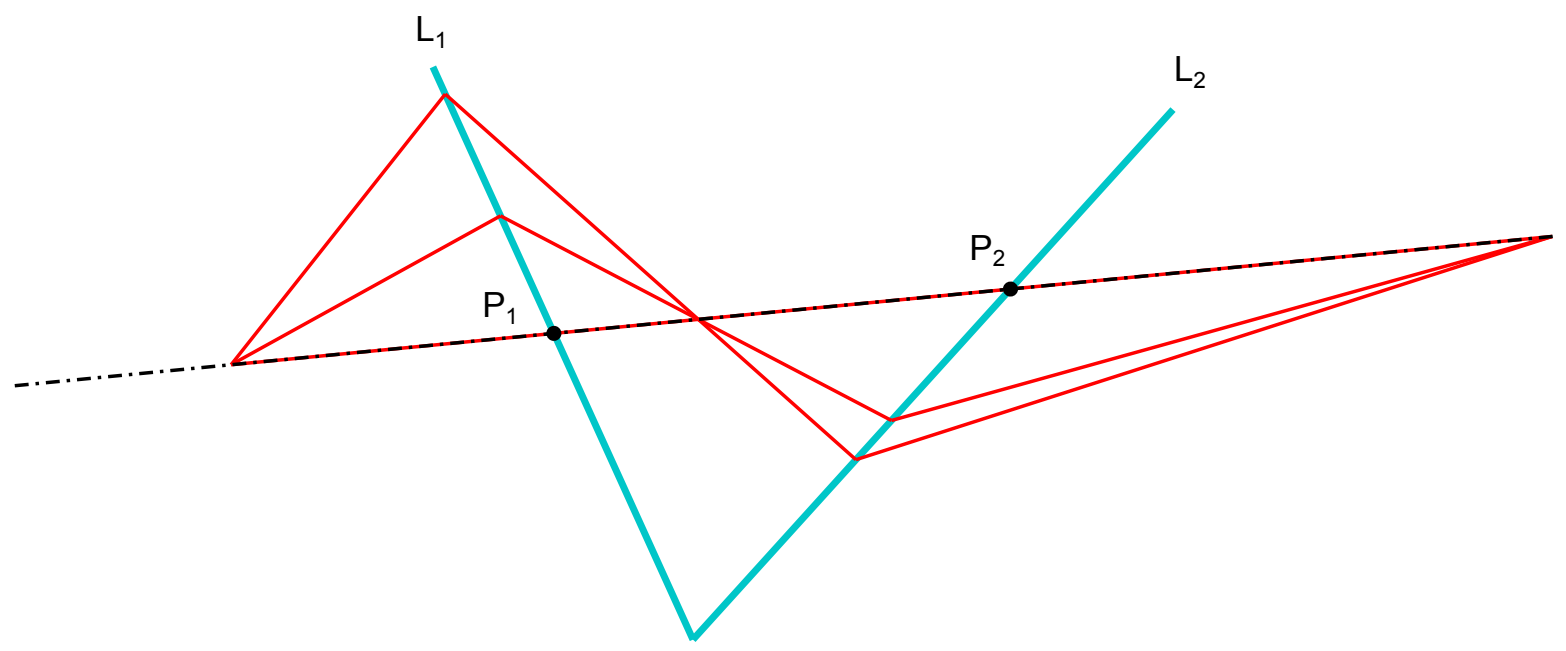

Figure 6. Optical axis of the skew-lens combionation. The optical axis (dash-dotted black line) passes through the principal points $\mathrm{P}_{1}$ and $\mathrm{P}_{2}$ of the individual lenses. It has the property that any point on the optical axis is imaged to another point on the optical axis.

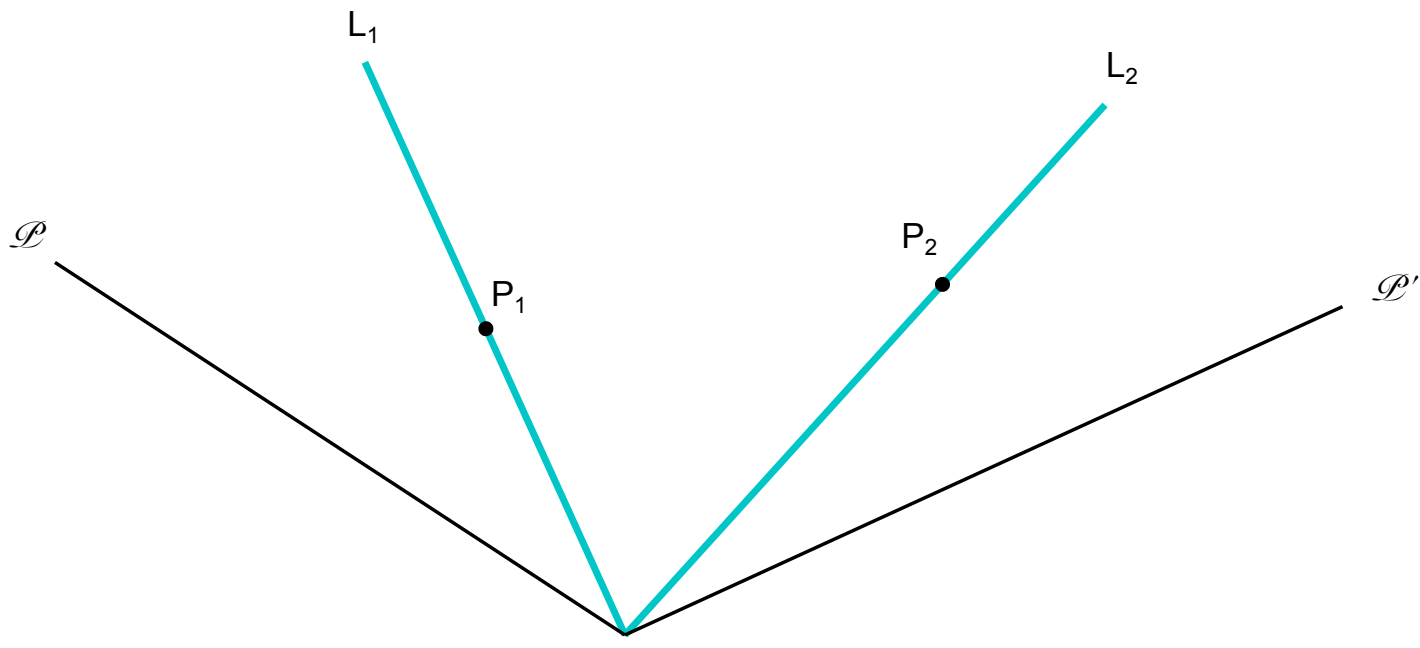

Figure 7. Principal planes of the lens combination. We define these as those transverse planes (solid black lines) through the line where the two lenses $\mathrm{L}_{1}$ and $\mathrm{L}_{2}$ intersect.

So far, we have made a number of definitions, shown in Fig. 8, and we have motivated these to an extent, but we have not shown that these definitions actually work. This can be done mathematically. We describe positions in object space in a skew coordinate system with axes in the direction of the optical axis, the direction of the line in which the two lens planes intersect, and the direction in an object-space transverse plane that is orthogonal to the lens-intersection line; and with its origin placed at the object-sided principal point, which is the point where the optical axis intersects the object-sided principal plane. We describe image-space positions in a skew coordinate system whose axes point in the direction of the optical axes, the direction of the lens intersection and a transverse direction perpendicular to the lens intersection, and whose origin is positioned at the image-space principal point. It can then be shown that the equations describing the mapping between object and image space performed by the lens combination in these coordinates is the familiar single-lens mapping. 


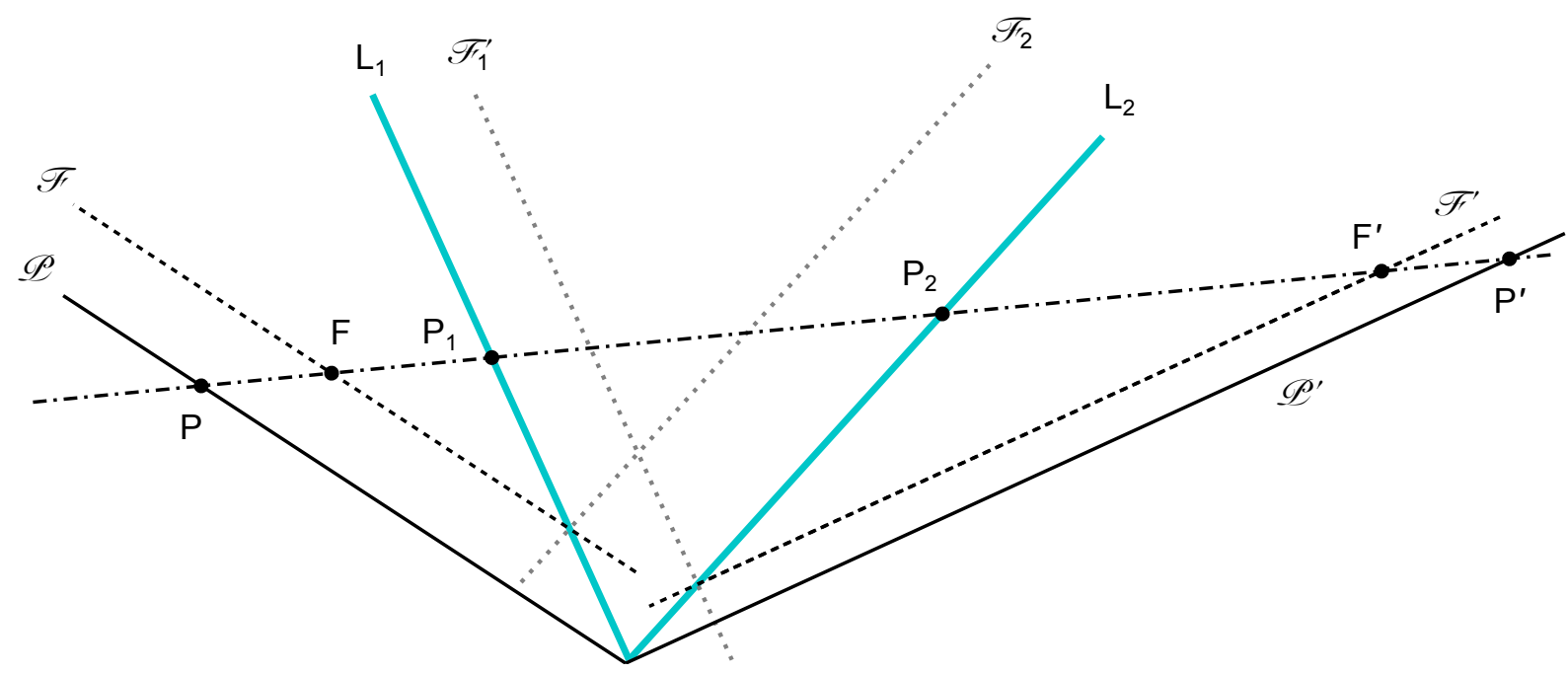

Figure 8. Cardinal planes and points of a combination of skew ideal thin lenses. As before, $\mathcal{P}$ and $\mathcal{P}^{\prime}$ (solid black lines) are the object- and image-sided principal planes, $\mathcal{F}$ and $\mathcal{F}^{\prime}$ (dashed black lines) are the object- and image-sided focal planes, and the dash-dotted line through the principal points of the individual lenses is the optical axis. We can also define the object- and image-sided principal points and focal points as those points where the corresponding plane intersect the optical axis.

\section{CONCLUSIONS}

We have generalised the concepts of optical axis, transverse planes, focal planes, and principal planes to skew combinations of ideal thin lenses. The definition of the transverse planes is at the same time the resolution of the telescopic-behaviour mystery mentioned in the introduction. Our definitions allow combinations of skew ideal thin lenses to be described as a single thin lens. This, in turn, should allow us to better understand transformation-optics devices consisting of ideal thin lenses.

Future work includes quantifying the agreement of our description with the behaviour of combinations of skew real lenses, and identifying situations in which our description works well.

\section{ACKNOWLEDGMENTS}

This work was supported by the UK's Engineering and Physical Sciences Research Council [grant numbers $\mathrm{EP} / \mathrm{K} 503058 / 1$ and $\mathrm{EP} / \mathrm{M} 010724 / 1]$.

\section{REFERENCES}

[1] J. Courtial, "Ray-optical refraction with confocal lenslet arrays," New J. Phys. 10, p. 083033, 2008.

[2] A. C. Hamilton and J. Courtial, "Generalized refraction using lenslet arrays," J. Opt. A: Pure Appl. Opt. 11, p. 065502, 2009.

[3] J. Courtial, "Standard and non-standard metarefraction with confocal lenslet arrays," Opt. Commun. 282, pp. 2634-2641, 2009.

[4] T. Maceina, G. Juzeliūnas, and J. Courtial, "Quantifying metarefraction with confocal lenslet arrays," Opt. Commun. 284, pp. 5008-5019, 2011.

[5] A. C. Hamilton and J. Courtial, "Metamaterials for light rays: ray optics without wave-optical analog in the ray-optics limit," New J. Phys. 11, p. 013042, 2009.

[6] J. Courtial and T. Tyc, "Generalised laws of refraction that can lead to wave-optically forbidden light-ray fields," J. Opt. Soc. Am. A 29, pp. 1407-1411, 2012.

[7] J. Courtial, "Geometric limits to geometric optical imaging with infinite, planar, non-absorbing sheets," Opt. Commun. 282, pp. 2480-2483, 2009. 
[8] S. Oxburgh and J. Courtial, "Perfect imaging with planar interfaces," J. Opt. Soc. Am. A 30, pp. 2334-2338, 2013.

[9] J. Courtial, S. Oxburgh, and T. Tyc, "Direct, stigmatic, imaging with curved surfaces," J. Opt. Soc. Am. A 32, pp. 478-481, 2015.

[10] C. Hembd-Sölner, R. F. Stevens, and M. C. Hutley, "Imaging properties of the Gabor superlens," J. Opt. A: Pure Appl. Opt. 1, pp. 94-102, 1999.

[11] S. Oxburgh, C. D. White, G. Antoniou, E. Orife, and J. Courtial, "Transformation optics with windows," Proc. SPIE 9193, p. 91931E, 2014.

[12] S. Oxburgh, C. D. White, G. Antoniou, E. Orife, T. Sharpe, and J. Courtial, "Large-scale, white-light, transformation optics using integral imaging," J. Opt. 18, p. 044009, 2016.

[13] G. J. Chaplain, G. Macauley, J. Bělín, T. Tyc, E. N. Cowie, and J. Courtial, "Ray optics of generalized lenses," J. Opt. Soc. Am. A 33, pp. 962-969, 2016.

[14] T. Tyc, S. Oxburgh, E. N. Cowie, G. J. Chaplain, G. Macauley, C. D. White, and J. Courtial, "Omnidirectional transformation-optics cloak made from lenses and glenses," J. Opt. Soc. Am. A 33, pp. 10321040, 2016.

[15] S. Oxburgh, T. Tyc, and J. Courtial, "Dr TIM: Ray-tracer TIM, with additional specialist capabilities," Comp. Phys. Commun. 185, pp. 1027-1037, 2014. 